\title{
Co-option of a default secretory pathway for plant immune responses
}

\author{
Chian Kwon ${ }^{1}$, Christina Neu ${ }^{1} \uparrow$, Simone Pajonk ${ }^{1}$, Hye Sup Yun ${ }^{1}$, Ulrike Lipka ${ }^{1,2} \uparrow$, Matt Humphry ${ }^{1}$, Stefan Bau ${ }^{1}$, \\ Marco Straus ${ }^{1}$, Mark Kwaaitaal ${ }^{1}$, Heike Rampelt ${ }^{2}$, Farid El Kasmi ${ }^{3}$, Gerd Jürgens ${ }^{3}$, Jane Parker ${ }^{1}$, Ralph Panstruga ${ }^{1}$, \\ Volker Lipka $^{1,2} \uparrow \&$ Paul Schulze-Lefert ${ }^{1}$
}

Cell-autonomous immunity is widespread in plant-fungus interactions and terminates fungal pathogenesis either at the cell surface or after pathogen entry. Although post-invasive resistance responses typically coincide with a self-contained cell death of plant cells undergoing attack by parasites, these cells survive pre-invasive defence. Mutational analysis in Arabidopsis identified PEN1 syntaxin as one component of two pre-invasive resistance pathways against ascomycete powdery mildew fungi ${ }^{1-3}$. Here we show that plasma-membrane-resident PEN1 promiscuously forms SDS-resistant soluble $\mathrm{N}$-ethylmaleimide sensitive factor attachment protein receptor (SNARE) complexes together with the SNAP33 adaptor and a subset of vesicle-associated membrane proteins (VAMPs). PEN1-dependent disease resistance acts in vivo mainly through two functionally redundant VAMP72 subfamily members, VAMP721 and VAMP722. Unexpectedly, the same two VAMP proteins also operate redundantly in a default secretory pathway, suggesting dual functions in separate biological processes owing to evolutionary co-option of the default pathway for plant immunity. The disease resistance function of the secretory PEN1-SNAP33-VAMP721/722 complex and the pathogen-induced subcellular dynamics of its components are mechanistically reminiscent of immunological synapse formation in vertebrates, enabling execution of immune responses through focal secretion.

Arabidopsis is immune to non-adapted powdery mildew fungi such as Blumeria graminis and Erysiphe pisi, which in nature colonize grass and pea species, respectively. This non-host resistance requires both pre- and post-invasive immune responses, which are under separate genetic control ${ }^{2}$. The former response engages PEN1 syntaxin, peroxisomal PEN2 $\beta$-glycosyl hydrolase and the plasmamembrane-resident PEN3 ABC transporter ${ }^{1-3}$. PEN2 and PEN3 act in the same pathway and are implicated in the cytoplasmic synthesis and transport of small antimicrobial compounds across the plasma membrane at attempted fungal entry sites, respectively ${ }^{2,3}$. PEN1 syntaxin acts in a second pathway and could, by analogy to known syntaxin functions in yeast and animals, either participate in vesicle fusion processes ${ }^{4}$ or modulate ion-channel activity through interactions with plasma-membrane-resident ion channels ${ }^{5}$. Genetic studies defy mechanistic interpretations but suggest direct or indirect PEN1 repressor activity in defence responses that are dependent on salicylic acid, as well as an overlapping function with the closely related syntaxin of plant $122(\text { SYP } 122)^{6}$.

Compared with largely resistant PEN1 wild type and severely defence-compromised pen1-1 null mutants, plants containing the pen1-3 allele allow intermediate B. graminis entry rates, indicating residual PEN1-3 resistance activity (Supplementary Fig. 1a). In the deduced PEN1-3 protein, a glycine residue is substituted by a glutamate in the SNARE domain ${ }^{1}$ (Supplementary Fig. 1b). Because this mutation affects a hydrophobic residue that is thought to stabilize interactions with other SNARE proteins in hetero-oligomeric SNARE complexes ${ }^{7}$ and is conserved in the SYP12 subfamily ${ }^{8}$, we hypothesized that PEN1-3 might be impaired in SNARE complex formation. Immunoblot analysis of leaf protein samples from wildtype, pen1-1 and pen1-3 plants with a PEN1 antiserum showed that monomeric PEN1-3 and wild-type PEN1 levels are indistinguishable and that PEN1-1 is undetectable (Fig. 1a), thus demonstrating that the amino-acid exchange in PEN1-3 does not affect protein stability. Notably, this analysis revealed small amounts of an SDS-resistant and heat-labile PEN1-containing complex in wild-type plants whose abundance increased upon fungal inoculation (Fig. 1a). This complex migrated at about 150 kilodaltons $(\mathrm{kDa})$ and was undetectable in pen1-3 plants (see over-exposed immunoblot; Fig. 1a). Because SDS resistance and heat sensitivity are typical features of exocytic ternary SNARE complexes in yeast and animals ${ }^{9}$, we concluded that in planta PEN1-3 either has a specific defect in SNARE complex formation or that PEN1-3 complex is unstable.

Binary target membrane $(\mathrm{t})$-SNARE complexes are typically formed by a syntaxin and SNAP25 at the plasma membrane during exocytosis ${ }^{4}$. Hence, we examined the expression of all three Arabidopsis SNAP25 homologues, SNAP29, SNAP30 and SNAP33, in leaves, the target tissue of powdery mildew fungi, by reverse transcriptase-polymerase chain reaction (RT-PCR) and detected exclusively SNAP33 transcripts (Supplementary Fig. 2a). Upon challenge with powdery mildew, both SNAP33 transcript and protein increased in abundance (Supplementary Figs 2a and 4a). SNAP33 assembled in planta in a pathogen-inducible SDS-resistant complex of similar size to the PEN1 complex and is capable of interacting in vitro with PEN1 (Supplementary Figs 2b and 3a). Analysis of transgenic Arabidopsis plants co-expressing functional fluorochromemarked yellow fluorescent protein (YFP)-SNAP33 and cyan fluorescent protein (CFP)-PEN1 fusion proteins revealed co-localization and focal accumulation of both proteins at the plasma membrane beneath incipient powdery mildew entry sites (Supplementary Fig. $2 \mathrm{c}-\mathrm{f}$ ). Together, these findings suggest that SNAP33 cooperates with PEN1 at the plasma membrane. However, lethality of snap33 mutants before flowering, associated with dwarfism, cytokinetic defects and necrotic leaf lesion formation, points to additional SNAP33 engagement(s) in essential cellular processes ${ }^{10}$.

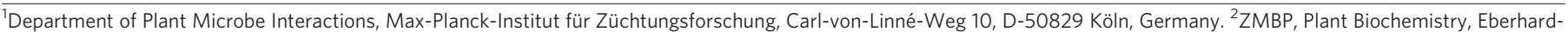

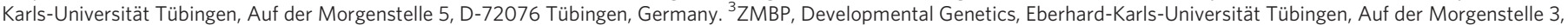

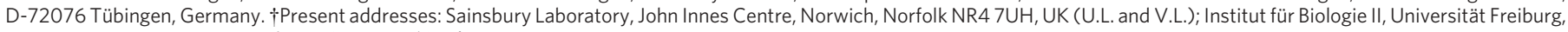
Sonnenstrasse 5, D-79104 Freiburg, Germany (C.N.). 
Exocytosis is completed by vesicle fusion with target membranes and this is thought to be driven by complex formation of t-SNAREs with vesicle (v)-SNAREs, also called vesicle-associated membrane proteins (VAMPs) ${ }^{4,11}$. The Arabidopsis genome harbours 12 VAMP encoding genes, which fall into two subfamilies ${ }^{8}$. The detection of
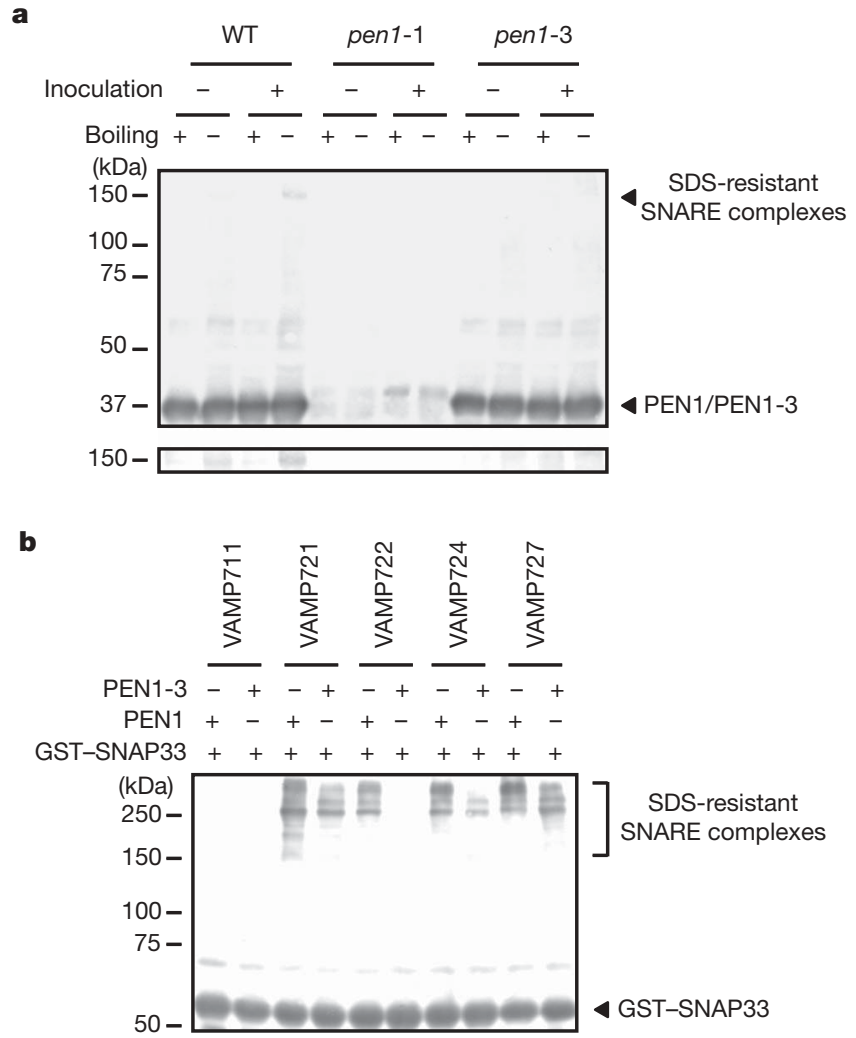

c

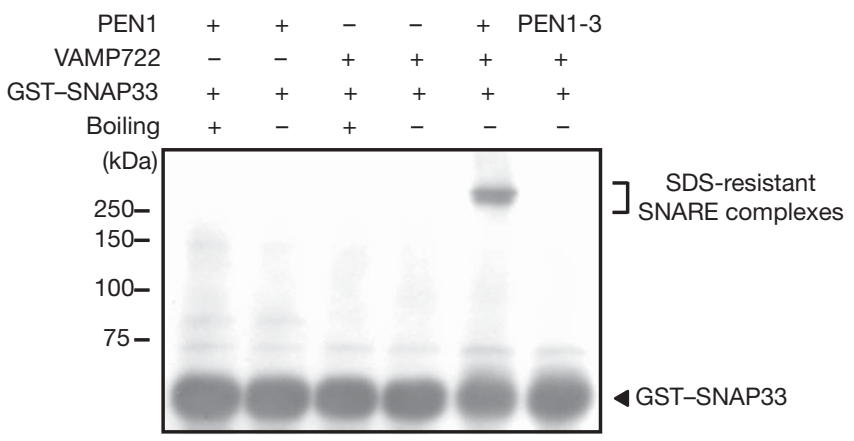

Figure 1 | PEN1-3 fails to form SDS-resistant SNARE complexes with

VAMP722. a, A pathogen-inducible and SDS-resistant PEN1-containing complex is undetectable in pen1-3 plants. Proteins were extracted from leaves of wild-type (WT), pen 1-1 or pen 1-3 plants. Samples were taken from healthy leaves or those challenged with $B$. graminis $24 \mathrm{~h}$ after spore inoculation. Proteins $(20 \mu \mathrm{g})$ were loaded on a $7.5 \%$ polyacrylamide gel and subjected to immunoblot analysis with anti-PEN1 antiserum. The lower panel shows a section of the same immunoblot corresponding to the PEN1containing complex after over-exposure. $\mathbf{b}$, Comparison of the SNARE complex-forming activity between PEN1 and PEN1-3. PEN1 or PEN1-3 $(2 \mu \mathrm{M})$ were incubated with equimolar GST-SNAP33 and the indicated VAMPs. Bound proteins were pulled down with glutathione-Sepharose 4B and eluted with sample buffer containing SDS. Eluted proteins were loaded on a $7.5 \%$ polyacrylamide gel and subjected to immunoblot analysis with anti-GST antibody. Note that the in vitro SNARE complexes migrate at a different size to the in vivo PEN1-containing complex because of the presence of epitope tag. c, All three SNARE proteins are required for the formation of the SDS-resistant complex. The indicated proteins $(2 \mu \mathrm{M})$ were incubated and SNARE complex formation was analysed as in $\mathbf{b}$. in planta PEN1-containing SNARE complex(es) prompted us to devise an in vitro systematic SNARE complex survey using purified Arabidopsis SNARE proteins that were expressed in Escherichia coli. We detected SDS-resistant SNARE complexes by incubating PEN1 and glutathione-S-transferase (GST)-SNAP33 bound to glutathione Sepharose 4B with various VAMPs. Sedimented bead-bound GSTSNAP33 was released and detected by immunoblot analysis using a GST antibody. GST-SNAP33 was able to form SDS-resistant and heat-sensitive complexes in the presence of PEN1 and each of four tested members of the VAMP72 subfamily, but not with VAMP711 (Fig. 1b). Barely detectable GST-SNAP33 complexes were seen in the presence of other VAMP71 subfamily members, VAMP712 and VAMP713 (not shown), suggesting that PEN1 associates preferentially with VAMP72 subfamily members. Importantly, when PEN1 was replaced by PEN1-3, complex formation was undetectable in the presence of VAMP722 and was reduced upon incubation with the other VAMP72 family members (VAMP721, VAMP724 and VAMP727; Fig. 1b). The presumed presence of all three SNARE proteins, PEN1, SNAP33 and VAMP722, in the SDS-resistant complex was validated by differential protein tagging ( $\mathrm{His}_{6}-\mathrm{PEN} 1$, GST-SNAP33 and HA-VAMP722) and immunoblot analysis using anti-His $_{5}$, anti-GST and anti-HA antibodies, respectively (Supplementary Fig. 3b, c). Pairwise association tests showed that PEN1 and VAMP722 do not associate alone under our experimental conditions and that PEN1-3 has a decreased affinity for SNAP33 (Supplementary Fig. 3a). Moreover, SDS-resistant complex formation required the presence of PEN1, SNAP33 and VAMP722 (Fig. 1c), demonstrating that all three SNARE proteins cooperate in vitro. This, and the lack of an in planta SDS-resistant PEN1-3 complex, identifies VAMP722 as potential major v-SNARE partner of PEN1. However, the residual PEN1-3 disease resistance activity to B. graminis (Supplementary Fig. 1a) suggests that either additional VAMP72 family members contribute to PEN1 function or that unstable PEN1-3SNAP33-VAMP722 complexes retain in planta residual activity.

To address this, we used a genetic approach and selected homozygous T-DNA insertion lines for VAMP712, VAMP714, VAMP721, VAMP722, VAMP723 and VAMP727. All T-DNA lines showed wild-type-like entry rates of $B$. graminis into leaf epidermal cells (not shown), indicating potential genetic redundancy. Because VAMP721 is most closely sequence-related to VAMP722, we aimed to isolate vamp721 vamp722 double mutant lines. We generated plants that were homozygous mutant for either VAMP721 or VAMP722 and heterozygous for the respective other gene and analysed the genotypes of their progeny from selfings (Supplementary Table 1). Among 667 progeny tested, however, there were no plants that were homozygous mutant for both genes. This suggests fully penetrant lethality of vamp721 vamp722 double mutants and essential as well as redundant wild-type gene functions in development. Moreover, closer inspection of the segregation ratios of progeny genotypes recovered from the two parental lines VAMP721 $1^{-1-}$ VAMP $722^{+/-}$and VAMP721 $1^{+/-}$VAMP $722^{-/-}$indicated a deviation from the expected 1:2 segregation, but this was statistically significant only for the latter $(P=0.027$; Supplementary Table 1$)$. This reveals an additional gametophytic activity that is normally not apparent because of the redundant VAMP721 and VAMP722 function in diploid plants. Interestingly, VAMP721 and VAMP722 appear to act in compensating pathways as VAMP722 transcript abundance was markedly increased in the homozygous vamp721 single mutant background. A similar though less dramatic compensatory VAMP721 transcript increase was found in homozygous vamp722 plants (Supplementary Figs $5 \mathrm{c}$ and 7a). Transgenic 35S::RNAi $i_{V A M P 722}$ gene silencing lines in which both VAMP721 and VAMP722 transcript levels were specifically and severely reduced grew normally for about two weeks. Thereafter, necrotic leaf lesions appeared and the plants exhibited an overall dwarf stature that was accompanied by a reduction of leaf mesophyll cell size of approximately $60 \%$ (Supplementary Fig. 4b-d). Because the same phenotype 
appears with the same timing in pen1 syp122 double mutants ${ }^{6,12}$, this might indicate overlapping VAMP721/VAMP722 and PEN1/SYP122 functions.

Next we generated transgenic Arabidopsis lines for conditional VAMP721 VAMP722 co-silencing using the ethanol-inducible alc system $^{13}$. Using low concentrations of ethanol vapour applied to two-week-old plants and subsequent challenge with B. graminis spores, we identified multiple lines in which fungal entry rates were significantly elevated in an inducer-dependent manner; that is, showing a pen 1 phenocopy (Fig. 2a and Supplementary Fig. 5a-c). Because elevated fungal entry was observed without recognizable pleiotropic plant growth effects and correlated with a reduction of VAMP721 and VAMP722 transcript levels (Supplementary Fig. 5c), this provides genetic evidence for a VAMP721/VAMP722 function in disease resistance that can be uncoupled from their essential role in development.

For direct in planta VAMP721/722 immunodetection we generated an anti-VAMP721/722 antibody using the cytosolic part of VAMP722 (VAMP722 $\Delta$ TM). This antibody specifically detected both VAMP721 and VAMP722 sharing 96\% identical amino-acid residues (Fig. 2b). Analysis of the steady-state level of both proteins in leaf extracts showed dependence on gene dosage and revealed that the relative amount of VAMP722 apparently greatly exceeds that of VAMP721 (Fig. 2b). We used protein extracts from transgenic plants expressing functional SNAP33-myc driven by a 2.6 kilobase (kb) native promoter sequence in a snap33 background ${ }^{10}$ for coimmunoprecipitation experiments with the anti-myc antibody. Anti-myc immune complexes contained both PEN1 and VAMP721/ VAMP722 (Fig. 2c). Likewise, immunoprecipitation experiments with extracts from transgenic plants expressing functional green
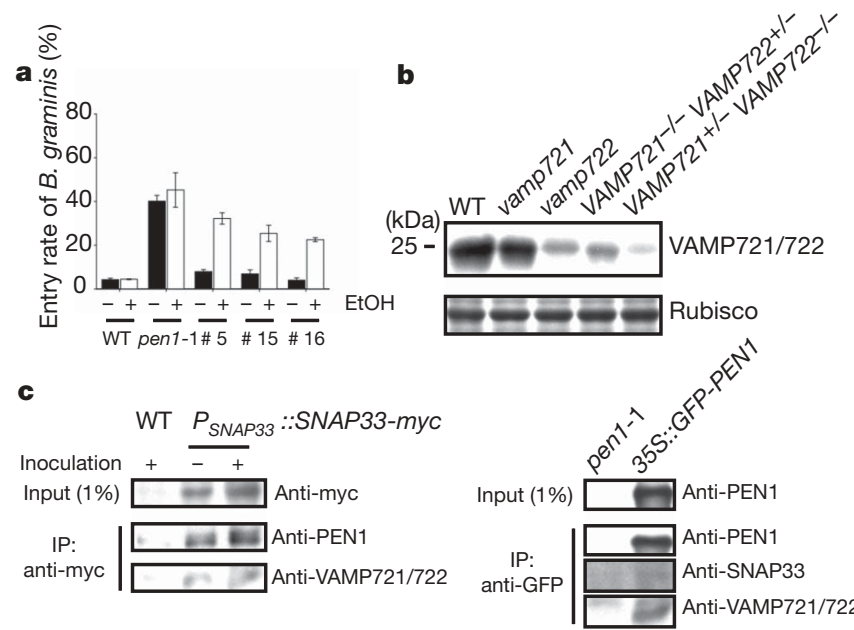

Figure 2 | VAMP721 and VAMP722 are required for PEN1-mediated immune responses. a, Compromised pre-invasion resistance after conditional silencing of VAMP721 and VAMP722 expression. The entry rate of $B$. graminis is increased in the alc::RNAi $i_{V A M P 722}$ silencing plants in an ethanol-dependent manner. Leaves of the indicated plants were harvested at $48 \mathrm{~h}$ post-inoculation with $B$. graminis spores and analysed by aniline blue staining and ultraviolet-light microscopy. Error bars indicate standard errors $(n=4)$. b, Gene-dosage-dependent control of VAMP721/722 steadystate levels. VAMP721/722 protein levels were analysed by leaf proteins extracts from the indicated plant genotypes by immunoblot using antiVAMP721/722 antibody. Note that the VAMP721/722 level in VAMP721 $1^{-/-}$VAMP $722^{+/-}$or VAMP721 $1^{+/-}$VAMP $722^{-/-}$plants is lower than in vamp 721 or vamp 722 plants, respectively. c, In planta interactions between PEN1, SNAP33 and VAMP721/722. Immunoprecipitation(IP) was performed with an anti-myc (left) or anti-GFP (right) antibody using protein extracts from the indicated plant genotypes. Leaf material was harvested $24 \mathrm{~h}$ after B. graminis spore inoculation or from healthy plants. Immune complexes were analysed by immunoblot using anti-PEN1, antiSNAP33 or anti-VAMP721/722 antibody. Protein extracts from wild-type or pen 1-1 plants were used as negative control. fluorescent protein (GFP)-PEN1 driven by the $35 \mathrm{~S}$ promoter in a pen1-1 background ${ }^{1}$ revealed in anti-GFP immune complexes the presence of SNAP33 and VAMP721/VAMP722 (Fig. 2c). Together this provides direct evidence for in planta PEN1-SNAP33VAMP721/VAMP722 ternary SNARE complexes.

To investigate the subcellular localization of VAMP722 in living plant cells in response to fungal attack, we generated transgenic plants expressing GFP fused to VAMP722 (GFP-VAMP722) under the control of $1.2 \mathrm{~kb}$ native $5^{\prime}$ regulatory VAMP722 sequences in the vamp722 background. These lines showed a pathogen-inducible accumulation of functional GFP-VAMP722, as indicated by immunoblot analysis of leaf protein extracts and restored resistance to fungal entry (Supplementary Figs $4 \mathrm{a}$ and $7 \mathrm{~b}$ ). Confocal microscopy revealed focal accumulation of GFP-fluorescent intracellular compartments in single attacked leaf epidermal cells directly beneath B. graminis appressoria (Fig. 3b). Whereas the polarized accumulation of GFP-VAMP722 is similar to the focal concentration of plasma-membrane-resident CFP-PEN1 $1^{12,14}$ and YFP-SNAP33 at incipient entry sites (Supplementary Fig. 2c-f), the former marks a different compartment(s). GFP-VAMP722-tagged structures differing in size were distinguishable (Fig. 3b). This is reminiscent of compound exocytosis in mammals in which vesicles fuse with each other
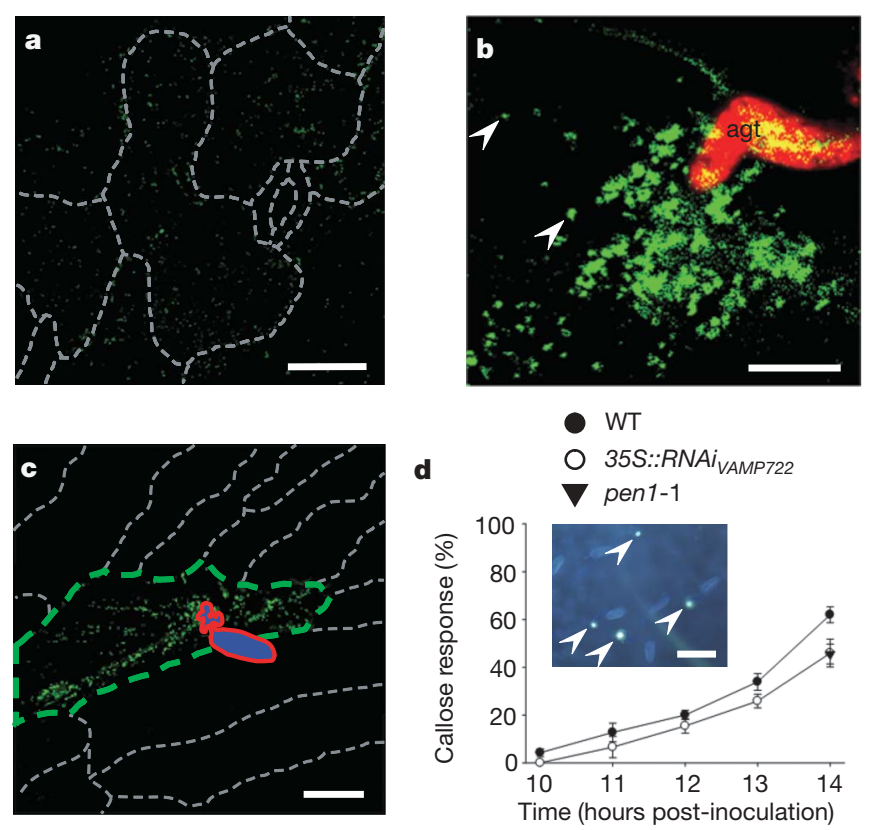

Figure 3 | Focal accumulation and directed movement of VAMP722containing intracellular compartments to powdery-mildew entry sites. a, Random distribution of GFP-tagged compartments in a cluster of epidermal pavement and stomata cells (grey dashed lines mark cell margins) of a healthy plant. The image combines stack signals of ten confocal sections at $1.91 \mu \mathrm{m}$ intervals. Scale bar, $25 \mu \mathrm{m}$. b, Focal accumulation of mobile punctuate structures labelled by GFP-VAMP722 (green) to a fungal entry site of a leaf epidermal cell. Note different sizes of the punctuate compartments (arrowheads). The picture was taken $15 \mathrm{~h}$ after inoculation with B. graminis spores (red). agt, appressorial germ tube. Scale bar, $10 \mu \mathrm{m}$. c, Cell-autonomous expression of GFP-VAMP722 and directed movement of GFP-fluorescent compartments to a fungal entry site on an epidermal midrib cell. The picture was taken $13 \mathrm{~h}$ after inoculation with $E$. pisi spores (red-lined blue). Note that the signal of GFP-VAMP722 is only detectable in the attacked cell (green dashed lines; grey dashed lines mark neighbouring epidermal cells). Scale bar, $40 \mu \mathrm{m}$. d, Retarded callose deposition in $35 S:: R N A i_{\text {vamp } 722}$ silencing plants. Callose deposition was visualized by aniline blue at the appressorial germ tubes (arrowheads in inset) and was determined at the indicated time points after B. graminis spore inoculation. Filled circle, wild type; open circle, $35 S:: R N A i_{\text {vamp } 722}$ silencing plants; filled triangle, pen 1-1. Error bars indicate standard errors $(n=3)$. Scale bar, $50 \mu \mathrm{m}$. 
before their fusion with the plasma membrane ${ }^{15}$ and/or represents aggregation of the punctuate compartments seen in healthy plants (Fig. 3a). In response to challenge with E. pisi, GFP-VAMP722 fluorescence at the leaf mid-vein region was detectable only in single attacked epidermal cells and characterized by a directed movement of GFP-VAMP722-tagged compartments to the fungal invasion site (Fig. 3c). In contrast, epidermal pavement cells of healthy unchallenged plants showed low abundance and random distribution of fluorescent structures (Fig. 3a). Collectively, these data are consistent with a pathogen-inducible VAMP722-mediated delivery of secretory compartments to incipient fungal entry sites. Pathogen-induced de novo cell-wall biosynthesis beneath fungal appressoria is delayed in pen1-1 plants compared with wild type $\mathrm{e}^{12}$. We investigated in timecourse experiments PMR4/GSL5-mediated callose formation ${ }^{16,17}$ as a marker of this process in wild type, pen1-1 and $35 S:: R N A i_{V A M P 722}$ transgenic lines in which VAMP721 and VAMP722 are constitutively co-silenced. The incidence of callose formation in the silencing lines was lower relative to wild type at all inspected time points and similar to pen1-1 plants (Fig. 3d). This hints to a common role for VAMP721/VAMP722 and PEN1 in focal secretion at fungal entry sites and is consistent with the hypothesis that PEN1 syntaxin acts through an authentic SNARE complex by association with VAMP721/VAMP722.

Barley HvROR2 and HvSNAP34 are orthologues of Arabidopsis PEN1 and SNAP33, and are known to restrict entry of B. graminis into epidermal cells of barley leaves ${ }^{1}$. We searched the HarvEST (http://harvest.ucr.edu/) barley EST database (assembly 31) and identified one unigene contig, Hv4993, that encodes a presumptive orthologue (termed HvVAMP721) of Arabidopsis VAMP721/
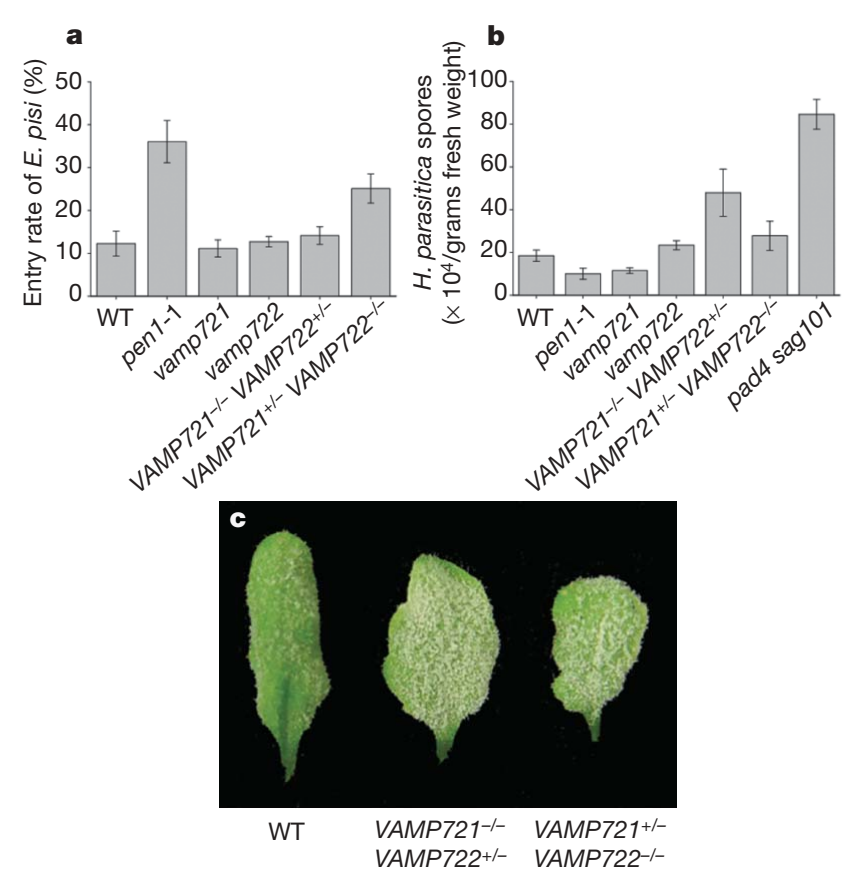

Figure 4 | Compromised pre- and post-invasion resistance by lowered gene dosage of VAMP721 and VAMP722. a, Increased entry rate of E. pisi in VAMP $721^{+/-} V A M P 722^{-/-}$plants. The entry rate was microscopically scored at seven days post-inoculation with $E$. pisi spores. Error bars indicate standard errors $(n=3)$. b, Hyper-susceptibility of VAMP721 ${ }^{-}$

VAMP $722^{+/-}$plants to $H$. parasitica. The susceptibility was analysed by counting conidiospore numbers at six days post-inoculation with $H$. parasitica spores. Error bars indicate standard errors $(n=3)$. c, Hypersusceptibility of both VAMP721 $1^{+/-}$VAMP722 $2^{-/-}$and VAMP721 VAMP722 $2^{+-}$plants to G. orontii. The susceptibility to G. orontii was macroscopically analysed with the indicated genotypes at ten days postinoculation. Note that inoculated leaves of VAMP721 $1^{+1-} V A M P 722^{-/-}$and VAMP $721^{-/-}$VAMP722 $2^{+/-}$plants are more densely covered with G. orontii mycelium and sporangiophores compared with wild type.
VAMP722 (Supplementary Fig. 6a). As in Arabidopsis, each of fluorescently labelled HvVAMP721, HvROR2 and HvSNAP34 accumulate at incipient powdery-mildew entry sites (not shown). Transient single-cell co-expression of fluorophore-tagged fusion proteins coupled with Förster resonance energy transfer analysis based on fluorescence lifetime imaging (FRET-FLIM) revealed pairwise in vivo interactions between HvROR2, HvSNAP34 and HvVAMP721 (Supplementary Fig. 6b), thereby supporting their capacity to cooperate in planta. Concentration of these barley SNARE proteins at fungal entry sites and in vivo interactions in the absence of the pathogen suggest that their dual function in immunity and cellular housekeeping is evolutionarily conserved.

We noted that the compensatory expression of VAMP722 or VAMP721 is abolished in VAMP721 $1^{-/-}$VAMP722 $2^{+/-}$and VAMP721 $1^{+/-}$VAMP722 $2^{-/-}$Arabidopsis plants (Supplementary Fig. 7a). Moreover, the transcript levels in these genotypes upon challenge with $B$. graminis are even lower than those in wild type and vamp721 or vamp722 single mutants (Supplementary Fig. 7a). This and the dependence on gene dosage of VAMP721/722 steadystate levels (Fig. 2b) prompted us to examine potentially impaired non-host disease resistance to $B$. graminis in VAMP $21^{+/-}$ VAMP722 $2^{-/}$and VAMP721 $1^{-/-}$VAMP722 $2^{+/}$genotypes. Only the former genotype allowed enhanced fungal entry into leaf epidermal cells, which was similar to the phenotype seen upon conditional co-silencing of VAMP721 and VAMP722 (Supplementary Fig. 5). Likewise, inoculation of the same genotypes with nonadapted E. pisi spores allowed elevated fungal entry rates only on VAMP721 $1^{+/-}$VAMP722 $2^{-/-}$plants (Fig. 4a). Thus, in the absence of the entry control function of VAMP722, VAMP721 becomes haplo-insufficient to both tested non-adapted powdery mildews. Intriguingly, VAMP721 $1^{-/-}$VAMP722 $2^{+/-}$plants were hypersusceptible to the virulent oomycete Hyaloperonospora parasitica (isolate Noco2), as indicated by elevated spore production at six days post-inoculation (Fig. 4b). This reveals VAMP722 haploinsufficiency in a homozygous vamp721 background and demonstrates that both VAMPs contribute to disease resistance against different pathogen classes. To test whether a similar resistance function restricts the growth of an adapted powdery mildew, we inoculated the respective genotypes with spores of Golovinomyces orontii.

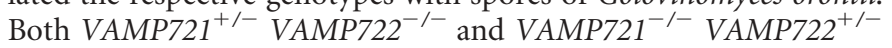
plants were hyper-susceptible to $G$. orontii (Fig. 4c and Supplementary Fig. 8a). This demonstrates that immune responses to non-adapted and adapted pathogens engage common secretory components and that VAMP721 and VAMP722 contribute to a wider range of defence responses than PEN1. However, bacterial growth of Pseudomonas syringae DC3000 was indistinguishable between the tested plant genotypes (Supplementary Fig. 8b). This and the disease resistance function of Nicotiana benthamiana NbSYP132 against P. syringa $e^{18}$, a syntaxin that belongs to a different SYP subfamily than PEN1 and SYP122, suggest that plants use a separate secretory pathway to restrict bacterial growth.

To our knowledge, we provide for the first time biochemical and genetic evidence for ternary SNARE complex functions in plants. Our findings show that PEN1-SNAP33-VAMP721/VAMP722 complexes are required for immune responses to powdery mildew/ oomycete pathogens, and that VAMP721 and VAMP722 act redundantly during development, thereby revealing dual functions in separate biological processes. Reduced gametophytic transmission of the vamp721 and/or vamp722 mutation, the lethality of vamp721 vamp722 double mutants, and plant dwarfism in $35 S:: R N A i_{V A M P 722}$ gene silencing lines (Supplementary Table 1 and Supplementary Fig. $4 \mathrm{~b}-\mathrm{d}$ ) probably reflect perturbation of an ancient default secretory pathway, which is essential for viability of plant cells. Both 35S::RNA $i_{V A M P 722}$ gene silencing lines and pen1 syp122 double mutants exhibit severe dwarfism ${ }^{6,12}$ (Supplementary Fig. 4c). However, unlike the lethal vamp721 vamp722 genotype, pen1 syp122 double mutants can be propagated as true breeding lines ${ }^{6,12}$. Thus, the 
default activity of VAMP721/VAMP722 cannot act solely through PEN1 and SYP122, but is likely to engage additional syntaxins. Similarly, the immune response function of VAMP721/VAMP722 cannot act solely through PEN1 because the VAMPs contribute to a wider range of defence responses than PEN1 (Fig. 4). Collectively, our findings suggest that Arabidopsis has co-opted the pre-existing VAMP721/VAMP722-dependent secretion apparatus for immune responses to pathogens. In one scenario, the default transport pathway could be re-used to deliver pathogen-inducible cargo. The broad-spectrum activity of the identified VAMPs against an ascomycete and oomycete parasite (Fig. 4) and the delay in pathogeninducible cell wall reinforcement in $35 S:: R N A i_{V A M P 722}$ transgenic lines (Fig. 3d) predict broadly effective antimicrobials ${ }^{19}$ and cell-wall components as candidate cargo.

Focal concentration of PEN1, SNAP33 and VAMP722 (Fig. 3b, c and Supplementary Fig. $2 \mathrm{c}-\mathrm{f}$ ) at pathogen entry sites and the resistance function of the corresponding SNARE complex provide a missing link to synthesize observations on seemingly diverse pathogen-induced cellular re-distribution events. This involves the disassembly and polar re-assembly of the actin cytoskeleton ${ }^{20}$, polar re-distribution of intracellular organelles ${ }^{21,22}$ including PEN2containing peroxisomes ${ }^{2}$, and the formation of lipid raft-like plasma membrane microdomains underneath pathogen entry sites ${ }^{14}$. This, and the requirement of the secretory SNARE complex for preinvasion resistance, are mechanistically strikingly similar to the formation of the immunological synapse in vertebrates for effective immune responses through focal secretion ${ }^{23,24}$. Cytotoxic T lymphocytes kill antigen-presenting cells by secreting perforin (also called cytolysin) and serine proteases upon recognition of non-self peptides presented by plasma-membrane-resident major histocompatibility complexes $^{25}$. In interactions with the non-adapted $B$. graminis and $E$. pisi powdery mildews, the resistance response restricts fungal entry into leaf epidermal cells. Because of the focal accumulation of PEN1, SNAP33 and VAMP722 beneath appressoria, we conclude that focal secretion terminates pathogenesis in a process that resembles the immunological synapse formation. Unlike powdery mildews that exclusively infect epidermal cells ${ }^{22}, H$. parasitica retrieves nutrients mainly from mesophyll cells by differentiation of ramified hyphae in the apoplastic space of mesophyll tissue ${ }^{21}$. Thus, the observed hyper-susceptibility to $G$. orontii and $H$. parasitica (Fig. 4b, c) implies impaired VAMP721/722 secretory activity both in epidermal and mesophyll cells. Activated T cells release different cargos by distinct secretory pathways: besides focal secretion through the immunological synapse, multi-directional secretion is used to promote inflammation ${ }^{26}$. Because adapted powdery mildews such as G. orontii or E. cichoracearum have overcome pre-invasion resistance $^{2,6}$, hyper-susceptibility to $G$. orontii could be partly due to reduced non-directional VAMP721/722 secretory activity from neighbouring cells.

\section{METHODS SUMMARY}

Plant material. All plants used for experiments were Arabidopsis Col-0, except pen 1-3 whose background was Arabidopsis Col-3 gll. All plants used were soil grown in growth chambers at $20-23^{\circ} \mathrm{C}$ with a $10 \mathrm{~h}$ photoperiod and a light intensity of about $150 \mu \mathrm{E} \mathrm{m}^{-2} \mathrm{~s}^{-1}$. For gene silencing experiments, two-weekold plants were used. The induction was performed in a closed chamber $(50 \mathrm{~cm} \times 30 \mathrm{~cm} \times 20 \mathrm{~cm})$ by ethanol vapour for 12 days. Gene silencing was periodically induced with alternating two-day intervals with and without ethanol. Total protein extracts were prepared from plant leaves by grinding the tissue in liquid nitrogen and suspending extracts in $1 \times \mathrm{PBS}(\mathrm{pH} 8.0)$ containing $1 \%$ Triton X-100. The solution was centrifuged and the supernatant used for further experiments.

To isolate homozygous vamp712 (At2g25340), vamp714 (At5g22360), vamp721 (At1g04750), vamp722 (At2g33120), vamp723 (At2g33110) and vamp727 (At3g54300) mutants, GABI_054H03, GABI_844B05, SALK_037273, SALK_119149, GABI_839H05 and GABI_060G05 lines were obtained from $\mathrm{ABRC}$ and GABI-Kat and analysed by PCR and DNA sequencing. To generate GFP-VAMP722 transgenic lines, GFP was fused in-frame to the amino (N) terminus of full-length VAMP722 and downstream of a $1.2 \mathrm{~kb}$ fragment of
VAMP722 5 ' regulatory sequence ( $\mathrm{P}_{\mathrm{VAMP} 722}:$ GFP-VAMP722). To generate transgenic plants expressing CFP-PEN1 and YFP-SNAP33, CFP or YFP was fused in-frame to the $\mathrm{N}$ terminus of full-length PEN1 or SNAP33, respectively, downstream of the 35S promoter (35S::CFP-PEN1 or 35S::YFP-SNAP33). Transgenic plants co-expressing both CFP-PEN1 and YFP-SNAP33 were obtained by crossing the above-mentioned transgenic lines. To generate VAMP721/VAMP722-silencing plants, complementary DNA (cDNA) corresponding to the cytoplasmic region of VAMP722 was inserted downstream of either the $35 \mathrm{~S}$ or alc promoter as inverted repeats. All transgenic plants were generated by transformation mediated by Agrobacterium tumefaciens.

Full Methods and any associated references are available in the online version of the paper at www.nature.com/nature.

\section{Received 23 October; accepted 12 December 2007.}

1. Collins, N. C. et al. SNARE-protein-mediated disease resistance at the plant cell wall. Nature 425, 973-977 (2003)

2. Lipka, V. et al. Pre- and postinvasion defenses both contribute to nonhost resistance in Arabidopsis. Science 310, 1180-1183 (2005).

3. Stein, M. et al. Arabidopsis PEN3/PDR8, an ATP binding cassette transporter, contributes to nonhost resistance to inappropriate pathogens that enter by direct penetration. Plant Cell 18, 731-746 (2006).

4. Ungar, D. \& Hughson, F. M. SNARE protein structure and function. Annu. Rev. Cell Dev. Biol. 19, 493-517 (2003)

5. Spafford, J. D. \& Zamponi, G. W. Functional interactions between presynaptic calcium channels and the neurotransmitter release machinery. Curr. Opin. Neurobiol. 13, 308-314 (2003).

6. Zhang, Z. et al. A SNARE-protein has opposing functions in penetration resistance and defense signalling pathways. Plant J. 49, 302-312 (2007).

7. Sutton, R. B., Fasshauer, D., Jahn, R. \& Brunger, A. T. Crystal structure of a SNARE complex involved in synaptic exocytosis at $2.4^{\circ} \mathrm{A}$ resolution. Nature 395 , 347-353 (1998).

8. Sanderfoot, A. A., Assaad, F. F. \& Raikhel, N. V. The Arabidopsis genome. An abundance of soluble $\mathrm{N}$-ethylmaleimide-sensitive factor adaptor protein receptors. Plant Physiol. 124, 1558-1569 (2000).

9. Hayashi, T. et al. Synaptic vesicle membrane fusion complex: action of clostridial neurotoxins on assembly. EMBO J. 13, 5051-5061 (1994).

10. Heese, M. et al. Functional characterization of the KNOLLE-interacting t-SNARE AtSNAP33 and its role in plant cytokinesis. J. Cell Biol. 155, 239-250 (2001).

11. Mayer, A. Membrane fusion in eukaryotic cells. Annu. Rev. Cell Dev. Biol. 18, 289-314 (2002).

12. Assaad, F. F. et al. The PEN1 syntaxin defines a novel cellular compartment upon fungal attack and is required for the timely assembly of papillae. Mol. Biol. Cell 15, 5118-5129 (2004).

13. Roslan, H. A. et al. Characterization of the ethanol-inducible alc gene-expression system in Arabidopsis thaliana. Plant J. 28, 225-235 (2001).

14. Bhat, R. A., Miklis, M., Schmelzer, E., Schulze-Lefert, P. \& Panstruga, R. Recruitment and interaction dynamics of plant penetration resistance components in a plasma membrane microdomain. Proc. Natl Acad. Sci. USA 102, 3135-3140 (2005)

15. Pickett, J. A. \& Edwardson, J. M. Compound exocytosis; mechanisms and functional significance. Traffic 7, 109-116 (2006).

16. Nishimura, M. T. et al. Loss of a callose synthase results in salicylic aciddependent disease resistance. Science 301, 969-972 (2003).

17. Jacobs, A. K. et al. An Arabidopsis callose synthase, GSL5, is required for wound and papillary callose formation. Plant Cell 15, 2503-2513 (2003).

18. Kalde, M., Nühse, T. S., Findlay, K. \& Peck, S. C. The syntaxin SYP132 contributes to plant resistance against bacteria and secretion of pathogenesis-related protein 1. Proc. Natl Acad. Sci. USA 104, 11850-11855 (2007).

19. van Loon, L. C., Rep, M. \& Pieterse, C. M. J. Significance of inducible defense-related proteins in infected plants. Annu. Rev. Phytopathol. 44, 135-162 (2006).

20. Kobayashi, Y. et al. Dynamic reorganization of microfilaments and microtubules is necessary for the expression of non-host resistance in barley coleoptile cells. Plant J. 11, 525-537 (1997)

21. Takemoto, D., Jones, D. A. \& Hardham, A. R. GFP-tagging of cell components reveals the dynamics of subcellular re-organization in response to infection of Arabidopsis by oomycete pathogens. Plant J. 33, 775-792 (2003).

22. Koh, S., Andre, A., Edwards, H., Ehrhardt, D. \& Somerville, S. Arabidopsis thaliana subcellular responses to compatible Erysiphe cichoracearum infections. Plant J. 44, 516-529 (2005)

23. Baluska, F., Volkmann, D. \& Menzel, D. Plant synapses: actin-based domains for cell-to-cell commmunication. Trends Plant Sci. 10, 106-111 (2005).

24. Kwon, C., Panstruga, R. \& Schulze-Lefert, P. Les liaisons dangereuses: immunological synapse formation in animals and plants. Trends Immunol. (in the press).

25. Lieberman, J. The ABCs of granule-mediated cytotoxicity: new weapons in the arsenal. Nature Rev. Immunol. 3, 361-370 (2003). 
26. Huse, M., Lillemeier, B. F., Kuhns, M. S., Chen, D. S. \& Davis, M. M. T cells use two directionally distinct pathways for cytokine secretion. Nature Immunol. 7 247-255 (2006).

Supplementary Information is linked to the online version of the paper at www.nature.com/nature.

Acknowledgements We thankE. Schmelzer, J. Bautor, A. Reinstädler and H. Häweker for technical assistance, $\mathrm{H}$. Thordal-Christensen for PEN1 antiserum, and Riyaz Bhat for initial FRET-FLIM measurements. This work was supported by funds from the Max Planck Society, the Deutsche Forschungsgemeinschaft (SFB670 and SPP1212) and the Bundesminsterium für Bildung und Forschung (GABI Nonhost Resistance
Consortium). C.K. was supported by a post-doctoral fellowship from the Korean Research Foundation (KRF) and M.H. by the Alexander von Humboldt Foundation.

Author Contributions C.K., R.P., V.L. and P.S.-L. designed all experiments. C.K., C.N., S.P., H.S.Y., U.L., M.H. S.B., M.S., M.K., H.R. and F.E.K. performed the experiments. C.K., V.L., G.J., R.P. and P.S.-L. analysed the data. C.K. and P.S.-L. wrote the paper.

Author Information Reprints and permissions information is available at www.nature.com/reprints. Correspondence and requests for materials should be addressed to V.L. (Volker.Lipka@sainsbury-laboratory.ac.uk), R.P.

(panstrug@mpiz-koeln.mpg.de) or P.S.-L. (schlef@mpiz-koeln.mpg.de). 


\section{METHODS}

Purification of recombinant proteins. Plasmid vector pGEX-6p-1(Amersham) was used for recombinant gene expression. Recombinant proteins were expressed in E. coli Rosetta (Invitrogen) and purified by affinity chromatography using glutathione-Sepharose 4B (Amersham). To obtain the proteins devoid of the GST moiety, the bead-bound proteins were digested with PreScission Protease (Amersham) and released proteins were collected. Protein concentration was measured by the Bio-Rad protein assay (Bio-Rad). DNA sequences corresponding to $\mathrm{His}_{6}{ }^{-}$or HA-tagged proteins were generated by PCR using 5 '-modified primers. Corresponding cDNAs were obtained from RIKEN.

Immunoblotting. Equimolar $(2 \mu \mathrm{M})$ purified recombinant proteins were incubated and interacting proteins were obtained by sedimenting GST-fused proteins bound to glutathione-Sepharose 4B. After intensive washing, the protein-bound beads were re-suspended in sample buffer and briefly centrifuged. The supernatant was boiled, separated on an acrylamide gel and probed with anti-GST or anti-His antibody. To visualize SDS-resistant SNARE complexes, supernatants were not boiled before loading. For SDS-resistant SNAREcomplex detection in plant extracts, equal amounts of total proteins $(20 \mu \mathrm{g})$ were separated on acrylamide gels and probed with anti-PEN1 antiserum or anti-myc antibody. To detect the presence of $\mathrm{His}_{6}-\mathrm{PEN} 1$, GST-SNAP33 and HAVAMP722 in SDS-resistant complexes, proteins $(4 \mu \mathrm{M})$ were incubated and separated on acrylamide gels. Gels were stained with Coomassie blue and the bands only detectable in unboiled samples were cut out. Subsequently, proteins were extracted in $1 \times$ PBS containing $1 \%$ Triton X-100 and probed with anti$\mathrm{His}_{5}$, anti-GST or anti-HA antibody to detect $\mathrm{His}_{6}$-PEN1, GST-SNAP33 or HAVAMP722, respectively. To detect VAMP721/722, a polyclonal anti-VAMP721/ 722 antiserum was raised in a rabbit using the cytosolic part of VAMP722 (VAMP722 $\Delta \mathrm{TM}$ ). VAMP722 $\Delta \mathrm{TM}$ and VAMP721 $\Delta \mathrm{TM}$ differ by only four amino acids.

In vivo interaction. To study interactions between PEN1, SNAP33 and VAMP721/722 in planta, co-immunoprecipitation and FRET assays were performed. For immunoprecipitation, the protein extracts from $P_{S N A P 33}:: S N A P 33-$ myc or 35S::GFP-PEN1 plants were pre-cleared with empty Protein A-agarose beads (Sigma-Aldrich) and incubated with anti-myc or anti-GFP antibody, respectively. After the addition of Protein A-agarose beads, the precipitates were analysed by anti-PEN1, anti-SNAP33 or anti-VAMP721/722 antibody. FRET analysis was performed with fluorescently labelled HvROR2, HvSNAP34 and HvVAMP722, as previously described ${ }^{27}$.

Gene expression. Total RNA was extracted from leaves with RNeasy Plant Mini Kit (Qiagen) according to the manufacturer's instructions. An equal amount of total RNA was used to analyse the expression of VAMP721 and VAMP722 by RT-PCR using the following primers: 5'-GAAGGCGCAAGTGTCAGA-3' and 5'-CAATAAAAGAGAAGGCACTAC-3' for VAMP721, and 5'-GGAACATTTCTTCCCGGC-3' and 5'-AGTACCAGCAATAGCAAC-3' for VAMP722. Radioactively labelled probes corresponding to the $3^{\prime}$ untranslated regions of VAMP721 or VAMP722 cDNAs were used for RNA gel blot analysis.

Pathogen inoculation and disease resistance scores. Three-week-old plants were used for inoculation with B. graminis, G. orontii or E. pisi conidiospores, two-week-old plants for infection with $H$. parasitica and five-week-old plants for infection with $P$. syringae. Resistance of plants to different pathogens was analysed as previously described ${ }^{1,2,28}$. Each set of inoculation experiments shown in Figs 2a, 3d, 4a, b and Supplementary Figs $1 \mathrm{a}, 7 \mathrm{~b}$ and $8 \mathrm{~b}$ was performed at least three times and produced similar results. To analyse entry rates of B. graminis in the conditional silencing plants, we counted 10-12 sectors from four leaves of two independent plants per experiment; each sector represented $0.65 \mu \mathrm{m}^{2}$.

27. Shen, Q. H. et al. Nuclear activity of MLA immune receptors links isolate-specific and basal disease-resistance responses. Science 315, 1098-1103 (2007).

28. Feys, B. J. et al. Arabidopsis senescence-associated gene101 stabilizes and signals within an enhanced disease susceptibility1 complex in plant innate immunity. Plant Cell 17, 2601-2613 (2005). 